\section{Trabalho e distúrbios psíquicos em professores da rede municipal de Vitória da Conquista, Bahia, Brasil}

\author{
Work and psychological distress among \\ public school teachers in Vitória da Conquista, \\ Bahia State, Brazil
}

Eduardo José Farias Borges dos Reis 1 Fernando Martins Carvalho 1 Tânia Maria de Araújo 2 Lauro Antônio Porto 3 Annibal Muniz Silvany Neto 1

\footnotetext{
1 Departamento

de Medicina Preventiva, Universidade Federal da Bahia, Salvador, Brasil.

2 Departamento de Saúde, Universidade Estadual de Feira de Santana, Feira de Santana, Brasil. 3 Fundação Nacional de Saúde, Salvador, Brasil.

Correspondência E. J. F. B. Reis Departamento de Medicina Preventiva, Universidade Federal da Bahia. Av. Reitor Miguel Calmon $s / n$, Salvador, $B A$ 40420-060, Brasil. eduardofreis@uol.com.br
}

\begin{abstract}
A cross-sectional study with all the teachers in the municipal school system in Vitória da Conquista, Bahia State, Brazil, investigated the association between work content (psychological demand and control over work) and the occurrence of minor psychiatric disorders (MPD) among teachers. The Karasek demand-control model was used to evaluate control over work. The Self-Report Questionnaire-20 was used to evaluate minor psychiatric disorders. MPD prevalence was $55.9 \%$ among the 808 teachers studied. Crude prevalence of MPD showed a positive and significant association with psychological demand and a negative and significant association with control over work. MPD prevalence was higher in teachers with highly demanding work, characterized by heavy demand and low control (PR = 1.74; 95\%CI: 1.44 2.10), and in those with active work, with heavy demand and low control $(P R=1.35$; 95\%CI: 1.13-1.61), as compared to teachers with lowdemand work (light demand and high control), after adjusting for confounders in a multiple logistic regression model. As a conclusion, teachers' mental health is strongly associated with their work content.
\end{abstract}

Mental Health; Working Conditions; Psychological Stress; Mental Disorders; Occupational Psychology

\section{Introdução}

A categoria docente é uma das mais expostas a ambientes conflituosos e de alta exigência de trabalho, tais como tarefas extra-classe, reuniões e atividades adicionais, problemas com alunos que chegam até ameaças verbais e físicas, pressão do tempo, etc. Esta situação estressante leva a repercussões na saúde física e mental e no desempenho profissional dos professores 1,2 . O impacto dos fatores estressantes sobre profissões que requerem condições de trabalho específicas, com grau elevado de relação com o público, como a do professor, tem sido estudado em outros países sob a denominação de Síndrome de Burned Out, ou Burnout que, no Brasil, recebeu a denominação de Síndrome do Esgotamento Profissional 3. Burnout seria uma síndrome de exaustão emocional e de atitudes cínicas e negativas dos profissionais em relação aos sentimentos dos indivíduos para os quais dirigem o seu trabalho, visto que os seus recursos emocionais estão esgotados. As conseqüências da Síndrome de Burnout são muito sérias para os vários setores relacionados a educação: professor, aluno, instituição (escola). As mudanças do papel do professor na sociedade refletem na prática de ensino e na saúde do professor, resultando em absenteísmo e solicitação de licença médica para tratamento de saúde, além da forma despersonalizada com que os professores começam a tratar os alunos 4,5. 
A bibliografia sobre a saúde do professor origina-se principalmente dos países centrais (Europa e América do Norte), destacando-se os estudos referentes ao estresse. No Brasil, pouco se tem feito para avaliar as repercussões do trabalho sobre a saúde do professor, cujos riscos são menos visíveis quando comparados a outros trabalhadores como metalúrgicos, petroquímicos etc. Recentemente, parcerias da Confederações de Trabalhadores em Estabelecimentos de Ensino (CONTEE) e da Confederação Nacional dos Trabalhadores na Educação (CNTE) com a Universidade Federal da Bahia (UFBA) e a Universidade de Brasília (UnB), respectivamente, produziram alguns estudos fundamentais. O estudo da UnB 6 sobre a saúde mental dos professores de primeiro e segundo graus em todo o país, abrangeu 1.440 escolas e 30 mil professores e revelou que $26,0 \%$ dos indivíduos estudados apresentavam exaustão emocional. Em Salvador, Bahia, uma pesquisa epidemiológica de corte transversal em 58 escolas e 573 professores de ensino privado, revelou um marcante processo de desgaste físico e mental, destacando-se a prevalência de distúrbios psíquicos menores (DPM) de 20,1\% 7 . Em professores da rede particular da cidade de Vitória da Conquista, Bahia, a prevalência de distúrbios psíquícos menores foi de $41,5 \% 8$.

O modelo demanda-controle de Karasek ${ }^{9}$ é um modelo bidimensional, oriundo da vertente de estudo sobre o estresse gerado no ambiente psicosocial do trabalho. Pretende relacionar o controle e a demanda forjada pela organização do trabalho no ambiente laboral com as repercussões sobre a estrutura psíquica e orgânica dos trabalhadores. Denomina-se como demanda psicológica ( $p$ sychological demand) aquelas situações de trabalho em que o trabalhador é exigido quanto à concentração, tempo para realização de tarefas, ritmo e volume etc. O controle do trabalho por parte do trabalhador (decision latitude), é considerado quanto a:

- Habilidades (skill discretion): criatividade, aprender coisas novas, tarefas diferentes, desenvolver habilidades especiais;

- Autoridade de decisão: liberdade de decidir como fazer as suas tarefas, opinar sobre o trabalho do grupo, influenciar na política gerencial.

O modelo demanda-controle prevê a avaliação simultânea de níveis de controle e de demanda, conformando situações de trabalho específicas. Ao combinar níveis de demanda e controle, diferentes situações de trabalho são constituídas:
- Alta exigência: combinando alta demanda e baixo controle;

- Trabalho ativo: combinando alta demanda e alto controle;

- Trabalho passivo: combinando baixa demanda e baixo controle;

- Baixa exigência: combinando baixa demanda e alto controle.

Posteriormente, o modelo demanda-controle incorporou uma outra dimensão psicológica, o suporte social, incluindo componentes de natureza coletiva, capazes de modificar as dimensões de ordem individuais da relação demanda-controle e saúde. O suporte social é considerado em dois subgrupos de questões: suporte da supervisão e suporte dos colegas de trabalho 9,10. Este modelo tem sido amplamente usado na área da saúde para avaliar agravos diversos, tais como doenças cardiovasculares, aborto, doenças osteomusculares e depressão 11 .

O modelo demanda-controle de Karasek é operacionalizado através de um instrumento conhecido como Job Content Questionnaire (JCQ). No Brasil, os dois estudos que avaliaram o desempenho do JCQ 12,13 relataram bons indicadores de validade e confiabilidade.

O presente estudo objetivou avaliar associação entre controle sobre o trabalho e demandas psicológicas e a ocorrência de distúrbios psíquicos menores entre professores da rede municipal de ensino fundamental de Vitória da Conquista.

\section{Metodologia}

Foi realizado um estudo epidemiológico de corte transversal na cidade de Vitória da Conquista. Com base na listagem das escolas da rede municipal de ensino, fornecida pela Secretaria Municipal de Educação, foi feito um censo abrangendo 219 escolas (186 na zona rural e 33 na zona urbana) e 20 creches. A rede municipal de ensino incorpora o ensino fundamental (pré-escola e os anos escolares de 1a a $8 \underline{a}$ série), abrangendo 1.058 professores. Foram incluídos neste estudo todos os professores em efetivo exercício profissional, independente do tipo de vínculo empregatício. Foram excluídos professores de educação física, xadrez, informática e línguas estrangeiras. Estes profissionais desempenham atividades de ensino com características bastante diferenciadas das atividades docentes mais tradicionais; em geral, envolvem menor carga horária e dinâmicas de ensino diferenciadas. 
Foi utilizado um questionário auto-aplicado para coleta de dados, com seis blocos de perguntas relacionadas a:

I. Identificação do professor;

II. Características gerais das atividades do professor na escola em que ele lecionava;

III. Aspectos psicossociais do trabalho (utilizando o JCQ na sua versão recomendada de 49 questões 14);

IV. Distúrbios psíquicos menores (utilizando o SRQ-20 - Self-Report Questionnaire 15);

V. Atividades domésticas.

Para a tradução para o português da versão do JCQ utilizada neste estudo, seguiu-se procedimentos estabelecidos pelo JCQ Center ${ }^{14}$. Controle e demanda foram as variáveis independentes principais desta investigação. Para a construção das escalas de demanda, controle e suporte social foram utilizados os critérios recomendados no manual do JCQ 14. Para dicotomizar a variável demanda usou-se a mediana como ponto de corte; para controle, usou-se o primeiro quartil como ponto de corte. A partir destas duas dimensões assim dicotomizadas, foram constituídas as quatro categorias: baixa exigência, trabalho ativo, trabalho passivo e alta exigência.

Os distúrbios psíquicos menores foram avaliados e classificados de acordo com os escores obtidos no SRQ-20 15. O ponto de corte utilizado para o estabelecimento de suspeição de distúrbios psíquicos foi de sete ou mais respostas positivas.

Diversas variáveis foram estudadas para investigação dos efeitos sobre a associação principal do estudo: sócio-demográficas (renda, idade, escolaridade, sexo, situação conjugal, ter filhos), hábitos saudáveis (prática de atividade física e de lazer), relacionadas ao trabalho docente (tempo de trabalho como professor, horas semanais de trabalho docente, número de turmas e alunos, número de colegas, vínculo de trabalho, modalidade de ensino, trabalhar em outra escola, exercício de outra atividade, zona de trabalho, tipo de escola, tempo gasto com deslocamento da escola, suporte social), atividades domésticas (indicador de sobrecarga doméstica, receber ajuda na atividade doméstica, número de habitantes na casa, cuidar das crianças, cuidar da limpeza da casa, cuidar da cozinha, lavar e passar roupa, pagar as contas da casa e fazer mercado e feira).

A variável suporte social foi codificada tendo como base a obtenção de apoio ou não da chefia ou dos colegas de trabalho, dividindo posteriormente pela mediana em duas categorias: baixo suporte e alto suporte.

O indicador de sobrecarga doméstica 16 é o somatório dos valores dos escores correspon- dentes a quatro tarefas domésticas básicas: cozinhar, limpar, lavar e passar roupa, ponderado pelo número de moradores na casa, exceto a própria $(\mathrm{N}-1)$. Cada uma das variáveis relativas às tarefas domésticas foi referida em uma escala ordinal, de 1 a 5 . Posteriormente, foi criada a variável ordinal sobrecarga doméstica, cujos tercis foram chamados de sobrecarga baixa, média e alta.

Para o processamento dos dados foi utilizado o programa SPSS. A população foi descrita segundo as variáveis sócio-demográficas, de trabalho docente e atividade doméstica. A medida de associação utilizada, entre demanda e controle e distúrbios psíquicos menores, foi a razão de prevalências e respectivos intervalos de confiança. A análise da presença de co-variáveis modificadoras de efeito ou confundimento foi realizada na primeira etapa da análise estratificada. Posteriormente, avaliaram-se os efeitos simultâneos das variáveis realizando a análise de regressão logística múltipla. Todas as co-variáveis foram incluídas na etapa de seleção das variáveis da análise de regressão logística múltipla.

$\mathrm{Na}$ análise estratificada e de regressão logística foram avaliadas a interação com as covariáveis referentes ao trabalho docente, ao trabalho doméstico e as características sóciodemográficas.

A análise estratificada teve como etapa inicial a escolha do grupo considerado de referência (grupo de baixa exigência) que teve sua prevalência comparada com a dos demais grupos de exposição. Prosseguindo, foram verificadas interações, observando-se se a razão de prevalência de cada nível de exposição de um estrato estava contido ou não no intervalo de confiança obtido para a razão de prevalência do outro estrato.

A regressão logística múltipla avaliou o efeito da associação principal, quando se ajustou concomitantemente pelas co-variáveis de interesse. Para a pré-seleção das co-variáveis observou-se as co-variáveis que apresentaram valor $\mathrm{p}$, obtido pelo teste de razão de verossimilhança, menor ou igual a 0,25 , em análises de regressão logística univariadas nas quais apenas a constante e uma variável de cada vez estavam contidas no modelo. A seguir, procedeuse à análise de regressão, incluindo a variável independente de interesse (modelo demandacontrole), as co-variáveis e os termos de interação de primeira ordem, utilizando-se o método “de trás para frente”, com reavaliação a cada etapa, para a seleção de variáveis.

Considerando que a prevalência de DPM estimada na população investigada foi elevada, 
distanciando-se dos parâmetros estimados para a OR (odds ratio), procedeu-se ao cálculo das estimativas de razões de prevalência (RP) e de seus respectivos intervalos de confiança, usando-se o procedimento do método Delta 17. Não foi realizado estudo piloto.

\section{Resultados}

Dos 967 professores elegíveis para pesquisa, registrou-se a perda de 159 (16,5\%), 80 (8,3\%) recusaram-se formalmente a participar do estudo e 79 (8,2\%) não foram localizados, incluídos aqui aqueles em licença médica. A população final do estudo foi de 808 educadores.

Os professores da rede municipal de Vitória da Conquista eram na maioria mulheres $(94,1 \%)$, casadas $(52,3 \%)$, com filhos $(58,5 \%)$ e com escolaridade de nível médio (67,5\%). A média de idade foi de 34,2 anos (desvio padrão $=8,5$ ) e a de número de filhos foi 2,0 (desvio padrão $=1,0$ ). Consumo de bebida alcoólica foi referido por $22,0 \%$ dos entrevistados e o hábito de fumar por $7,3 \%$. A realização de atividades domésticas foi referida por $86,7 \%$ da população: $95,9 \%$ entre as mulheres e $77,8 \%$ entre os homens. Sobrecarga doméstica alta foi encontrada para $32,8 \%$ das mulheres, e no caso dos homens, somente para $2,3 \%$.

O tempo médio de trabalho dos professores da rede municipal de Vitória da Conquista foi de $10,4( \pm 6,7)$ anos, com $85,2 \%$ deles com vínculo de trabalho estável (efetivos e concursados) e apenas $14,8 \%$ mantinham contrato precário (provisório). A modalidade de ensino mais freqüente foi a Fundamental I (1a à 4a séries), envolvendo $64,7 \%$ dos entrevistados. A média de turmas por professor foi $2,4,( \pm 2,1)$ com média de 29,4 $( \pm 7,6)$ alunos por sala de aula. A carga horária média encontrada em todas as escolas foi 38,8 $( \pm 11,1)$ horas por semana. Cerca de um terço dos professores $(32,1 \%)$ trabalhavam em uma outra escola, além daquela na qual ele foi entrevistado. Além das atividades de ensino, 5,8\% dos professores desenvolviam outra atividade remunerada fora da esfera da docência. Na zona urbana, trabalhavam 55,8\% dos professores. A renda mensal média dos professores da rede municipal de Vitória da Conquista foi de $\mathrm{R} \$ 477,00 \pm 203$, o que correspondia na época da pesquisa a US\$186,00 \pm 79 .

Dos 808 professores, $711(88,0 \%)$ responderam a todas as perguntas do SRQ-20. Distúrbios psíquicos menores ( $\geq 7$ respostas positivas dentre as 20 do SRQ-20) foram encontrados em $55,9 \%$ dos professores; sendo $57,2 \%$ nas mulheres e $34,0 \%$ nos homens.
As questões do SRQ-20 com maior freqüência de respostas afirmativas foram: "sente-se nervoso, tenso ou preocupado" (78,1\%), "você se cansa com facilidade" (59,8\%), "assusta-se com facilidade” (59,2\%), "tem sensações desagradáveis no estômago" (51,8\%), "tem dores de cabeça freqüentemente" (51,6\%) e "tem se sentido triste ultimamente" (49,1\%). As questões menos apontadas foram àquelas classificadas dentro do grupo de pensamentos depressivos (Tabela 1).

A Tabela 2 mostra que a prevalência de DPM estava estatisticamente associada $(\mathrm{p}<0,05)$ com 23 variáveis pesquisadas. Por outro lado, a prevalência de DPM não estava estatisticamente associada com 14 variáveis investigadas: idade ( $<26$; $\geq 26$ anos), escolaridade ( $\leq 20$ grau; $>$ 2o grau); estado conjugal (ter; não ter companheiro), ter filhos (sim; não), renda mensal (< R\$ 360,00; $\geq \mathrm{R} \$ 360,00)$, realizar atividade física (sim; não), ter atividade de lazer (sim; não), tipo de escola (isolada; círculo), número de modalidades de ensino $(1 ;>1)$, número de alunos na escola do estudo $(<25 ; \geq 25)$, exercer outra função na escola (sim; não), tempo de deslocamento para a escola $(<2 \mathrm{~h} ; \geq 2 \mathrm{~h})$, trabalhar em outra escola (sim; não) e trabalhar em outra atividade além da docente (sim; não).

Professores com distúrbios psíquicos menores apresentaram carga horária semanal em sala de aula significantemente maior $(\mathrm{p}<0,0001)$ que aqueles sem DPM: 29,6 $\pm 10,6$ versus $26,7 \pm$ 10,5 , respectivamente.

A grande maioria dos itens do JCQ esteve associados estatisticamente $(\mathrm{p}<0,05)$ com $o$ diagnóstico de DPM. Dez dentre as 13 questões relacionadas à demanda (Tabela 3), 10 dentre as 14 questões relacionadas ao controle (Tabela 4) e todas as 12 questões relacionadas a suporte social estavam associadas estatisticamente com DPM (Tabela 5).

A prevalência de DPM foi significantemente mais elevada nos professores com alta demanda (RP = 1,39; IC95\%: 1,21-1,59) (Tabela 3), baixo controle ( $\mathrm{RP}=1,22$; IC95\%: 1,06-1,41) (Tabela 4) e baixo suporte social $(\mathrm{RP}=1,27$; IC95\%: 1,13-1,44) (Tabela 5).

A prevalência bruta de DPM foi mais elevada no grupo de professores com trabalho de alta exigência $(77,8 \%)$, seguido de trabalho ativo $(62,4 \%)$, trabalho passivo $(51,8 \%)$ e, por último, baixa exigência $(45,1 \%)$. RP brutas e respectivos IC95\%, tomando como referência o grupo baixa exigência, são apresentadas na Tabela 6 .

Além da variável independente principal (demanda-controle), as seguintes variáveis foram analisadas: sexo, vínculo de trabalho, escolaridade, idade, situação conjugal, número 
Freqüência de respostas afirmativas para as perguntas do Self-Reporting Questionnaire-20 (SRQ-20), em professores da rede municipal de ensino de Vitória da Conquista, Bahia, Brasil, 2001.

\begin{tabular}{|c|c|c|}
\hline Fatores do SRQ-20 & $\mathbf{N}$ & $\%$ \\
\hline \multicolumn{3}{|l|}{ Diminuição da energia } \\
\hline Você se cansa com facilidade & 796 & 59,8 \\
\hline Tem dificuldade para tomar decisões & 794 & 46,5 \\
\hline Sente-se cansado o tempo todo & 791 & 40,6 \\
\hline Encontra dificuldade de realizar, com satisfação, suas tarefas diárias & 793 & 35,8 \\
\hline Tem dificuldade de pensar com clareza & 796 & 34,7 \\
\hline Seu trabalho diário Ihe causa sofrimento & 793 & 16,3 \\
\hline \multicolumn{3}{|l|}{ Sintomas somáticos } \\
\hline Tem sensações desagradáveis no estômago & 792 & 51,8 \\
\hline Tem dores de cabeça freqüentemente & 799 & 51,6 \\
\hline Dorme mal & 793 & 45,0 \\
\hline Tem má digestão & 789 & 44,1 \\
\hline Tem tremores nas mãos & 795 & 28,6 \\
\hline Tem falta de apetite & 793 & 28,2 \\
\hline \multicolumn{3}{|l|}{ Humor depressivo/ansioso } \\
\hline Sente-se nervoso, tenso ou preocupado & 795 & 78,1 \\
\hline Assusta-se com facilidade & 799 & 59,2 \\
\hline Tem se sentido triste ultimamente & 796 & 49,1 \\
\hline Tem chorado mais do que de costume & 796 & 29,9 \\
\hline \multicolumn{3}{|l|}{ Pensamentos depressivos } \\
\hline Tem perdido o interesse pelas coisas & 789 & 23,2 \\
\hline É incapaz de desempenhar um papel útil em sua vida & 794 & 12,6 \\
\hline Você se sente pessoa inútil em sua vida & 792 & 6,9 \\
\hline Tem tido idéia de acabar com a vida & 797 & 5,0 \\
\hline
\end{tabular}

de turmas, número de alunos, zona em que trabalho, trabalhar em outra escola, horas em sala de aula em todas as escolas, exercer outra atividade remunerada além de professor, renda, modalidade de ensino, ter atividade de lazer, realizar atividade física, ter suporte social no trabalho, ter filhos, sobrecarga doméstica, recebimento de ajuda no trabalho doméstico. Permaneceram no modelo selecionado, demonstrando estarem independentemente associadas à prevalência de DPM: sexo (feminino), vínculo de trabalho (estável), zona em que trabalha (urbana), horas em sala de aula em todas as escolas $(\geq 40)$ e recebimento de ajuda no trabalho doméstico (não receber). Após os procedimentos para seleção, nenhum termo de interação permaneceu no modelo final da regressão logística. Observou-se que as razões de prevalência e os níveis de significância estatística, obtidas após o ajuste simultâneo na análise de regressão logística por todas as variáveis con- fundidoras, diferiram ligeiramente das RP brutas para a associação entre DPM e conteúdo do trabalho (grupos do modelo demanda-controle). Assim, professores expostos a trabalho de alta exigência (alta demanda e baixo controle) apresentaram prevalência 1,74 vez mais elevada de distúrbios psíquicos menores do que trabalhadores em situações de baixa exigência (baixa demanda, alto controle), após ajuste pelo efeito das diversas co-variáveis. Professores em regime de trabalho ativo também apresentaram prevalências de distúrbios psíquicos menores significativamente mais elevados que aqueles em baixa exigência. Os professores com trabalho passivo apresentaram prevalência de DPM mais elevada que professores com trabalho de alta exigência, mas o limite inferior do IC95\% foi menor que a unidade. 
Razões de prevalência (RP) e IC95\% para a associação de distúrbios psíquicos menores (DPM) com características sócio-demográficas, hábitos de vida, características do trabalho docente, atividades domésticas e em professores da rede municipal de ensino de Vitória da Conquista, Bahia, Brasil, 2001.

\begin{tabular}{|c|c|c|c|c|c|}
\hline \multirow[t]{2}{*}{ Fatores de risco } & \multicolumn{5}{|c|}{ SRQ-20 } \\
\hline & $\mathrm{N}$ & $n$ & $\%$ & $\mathrm{RP}$ & IC95\% \\
\hline \multicolumn{6}{|l|}{ Sexo } \\
\hline Feminino & 741 & 424 & 57,2 & 1,68 & $1,12-2,51$ \\
\hline Masculino & 47 & 16 & 34,0 & & \\
\hline \multicolumn{6}{|l|}{ Número de filhos } \\
\hline$\leq 2$ & 335 & 208 & 62,1 & 1,21 & $1,07-1,36$ \\
\hline$\geq 3$ & 466 & 240 & 51,5 & & \\
\hline \multicolumn{6}{|c|}{ Tem atividade de lazer } \\
\hline Não & 500 & 299 & 59,8 & 1,20 & $1,04-1,38$ \\
\hline Sim & 264 & 132 & 50,0 & & \\
\hline \multicolumn{6}{|l|}{ Zona de trabalho } \\
\hline Urbano & 447 & 272 & 60,9 & 1,22 & $1,07-1,39$ \\
\hline Rural & 353 & 176 & 49,9 & & \\
\hline \multicolumn{6}{|c|}{ Vínculo de trabalho } \\
\hline Estável & 660 & 393 & 59,5 & 1,69 & $1,31-2,17$ \\
\hline Precário & 116 & 41 & 35,3 & & \\
\hline \multicolumn{6}{|c|}{ Tempo como professor (anos) } \\
\hline$\geq 5$ & 627 & 366 & 58,4 & 1,24 & $1,04-1,47$ \\
\hline$\leq 4$ & 174 & 82 & 47,1 & & \\
\hline \multicolumn{6}{|c|}{$\begin{array}{l}\text { Carga horária em sala de aula } \\
\text { na escola de estudo }\end{array}$} \\
\hline$\geq 25$ & 458 & 280 & 61,1 & 1,25 & $1,10-1,42$ \\
\hline$\leq 24$ & 343 & 168 & 49,0 & & \\
\hline \multicolumn{6}{|c|}{ Carga horária na escola de estudo } \\
\hline$\geq 40$ & 490 & 294 & 60,0 & 1,21 & $1,06-1,38$ \\
\hline$\leq 39$ & 311 & 154 & 49,5 & & \\
\hline \multicolumn{6}{|c|}{ Carga horária em todas as escolas } \\
\hline$\geq 40$ & 617 & 362 & 58,7 & 1,26 & $1,05-1,51$ \\
\hline$\leq 39$ & 152 & 71 & 46,7 & & \\
\hline \multicolumn{6}{|c|}{$\begin{array}{l}\text { Carga horária em sala de aula } \\
\text { em todas as escolas }\end{array}$} \\
\hline$\geq 40$ & 436 & 267 & 61,2 & 1,23 & $1,08-1,41$ \\
\hline$\leq 39$ & 312 & 155 & 49,7 & & \\
\hline \multicolumn{6}{|c|}{ Carga horária em todos os empregos } \\
\hline$\geq 40$ & 626 & 369 & 58,9 & 1,31 & $1,08-1,58$ \\
\hline$\leq 39$ & 144 & 65 & 45,1 & & \\
\hline \multicolumn{6}{|c|}{ Número de turmas na escola de estudo } \\
\hline$\leq 3$ & 636 & 369 & 58,0 & 1,21 & $1,02-1,44$ \\
\hline$\geq 2$ & 165 & 79 & 47,9 & & \\
\hline \multicolumn{6}{|c|}{$\begin{array}{l}\text { Número de colegas trabalho } \\
\text { na escola do estudo }\end{array}$} \\
\hline$\geq 20$ & 338 & 207 & 61,2 & 1,17 & $1,04-1,33$ \\
\hline$\leq 19$ & 420 & 219 & 52,1 & & \\
\hline
\end{tabular}

(continua) 


\begin{tabular}{|c|c|c|c|c|c|}
\hline \multirow[t]{2}{*}{ Fatores de risco } & \multicolumn{5}{|c|}{ SRQ-20 } \\
\hline & $\mathrm{N}$ & $\mathrm{n}$ & $\%$ & $\mathrm{RP}$ & IC95\% \\
\hline \multicolumn{6}{|l|}{ Cuidar da cozinha } \\
\hline Sim & 299 & 183 & 61,2 & 1,15 & $1,01-1,30$ \\
\hline Não & 487 & 260 & 53,4 & & \\
\hline \multicolumn{6}{|l|}{ Cuidar da limpeza } \\
\hline Sim & 327 & 204 & 62,4 & 1,20 & $1,06-1,35$ \\
\hline Não & 461 & 240 & 52,1 & & \\
\hline \multicolumn{6}{|l|}{ Lavar roupa } \\
\hline Sim & 310 & 194 & 62,6 & 1,20 & $1,06-1,36$ \\
\hline Não & 478 & 249 & 52,1 & & \\
\hline \multicolumn{6}{|l|}{ Passar roupa } \\
\hline Sim & 294 & 185 & 62,9 & 1,20 & $1,06-1,36$ \\
\hline Não & 485 & 255 & 52,6 & & \\
\hline \multicolumn{6}{|c|}{ Sobrecarga doméstica } \\
\hline Média/alta & 485 & 294 & 60,6 & 1,16 & $1,01-1,33$ \\
\hline Baixa & 251 & 131 & 52,2 & & \\
\hline \multicolumn{6}{|c|}{ Sobrecarga doméstica } \\
\hline Alta & 228 & 147 & 64,5 & 1,23 & $1,06-1,44$ \\
\hline Baixa & 251 & 131 & 52,2 & & \\
\hline \multicolumn{6}{|l|}{ Pagar conta } \\
\hline Sim & 348 & 216 & 62,1 & 1,19 & $1,05-1,34$ \\
\hline Não & 437 & 228 & 52,2 & & \\
\hline \multicolumn{6}{|c|}{ Receber ajuda em casa } \\
\hline Sim & 222 & 143 & 64,4 & 1,17 & $1,03-1,32$ \\
\hline Não & 518 & 286 & 55,2 & & \\
\hline \multicolumn{6}{|c|}{ Cuidar de criança(s)* } \\
\hline Sim & 193 & 126 & 65,3 & 1,27 & $1,11-1,46$ \\
\hline Não & 441 & 226 & 51,2 & & \\
\hline \multicolumn{6}{|c|}{ Fazer mercado/feira } \\
\hline Sim & 341 & 214 & 62,8 & 1,21 & $1,07-1,36$ \\
\hline Não & 445 & 231 & 51,9 & & \\
\hline
\end{tabular}

* Foram excluídos da análise 137 professores que referiram não ter crianças.

\section{Discussão}

A elevada prevalência de 55,9\% para os distúrbios psíquicos menores nos professores da rede municipal de Vitória da Conquista denota uma situação de saúde mental preocupante. Esta prevalência é muito superior às obtidas em outros estudos que investigaram a saúde dos professores que variaram de 18,0 a $24,2 \%$ $7,18,19,20,21$. Um estudo realizado em professores da rede privada de ensino em Vitória da Conquista 8 , encontrou a prevalência de $41,5 \%$. Mesmo comparado a outras categorias ocupacionais, o presente estudo encontrou com maior proporção de DPM: 19,0\% em metalúrgicos 22 , 20,0 a $24,0 \%$ em trabalhadores de processa- mentos de dados $23,20,8 \%$ em trabalhadores de hospital 24, agricultores rurais 25 e 33,3\% entre trabalhadoras de enfermagem 10 . Em um estudo populacional domiciliar nos bairros de Olinda, Pernambuco 26, o SRQ foi positivo na proporção de $35,0 \%$, resultado também menor do que o obtido em nosso estudo.

A grande maioria das características avaliadas pelo JCQ estavam fortemente associadas com a prevalência de DPM. Trabalho repetitivo, indicador de controle, e ritmo acelerado de trabalho, indicador de demanda psicológica, também estavam estatisticamente associados com DPM em professores de Salvador 7. O estudo realizado entre professores da rede particular de ensino de Vitória da Conquista 8 en- 
Razões de prevalência (RP) e IC95\% para a associação entre distúrbios psíquicos menores e questões relacionadas a demanda do Job Content Questionnaire (JCQ), nos professores da rede municipal de ensino de Vitória da Conquista, Bahia, Brasil, 2001.

\begin{tabular}{|c|c|c|c|}
\hline \multirow[t]{2}{*}{ Questões do JCQ } & \multicolumn{3}{|c|}{ SRQ-20 } \\
\hline & $\mathrm{N}$ & $\mathrm{RP}$ & IC95\% \\
\hline \multicolumn{4}{|l|}{ Demanda no trabalho } \\
\hline Alta demanda & 371 & 1,39 & $1,21-1,59$ \\
\hline Baixa demanda & 360 & 1,00 & - \\
\hline \multicolumn{4}{|l|}{ Demandas psicológicas } \\
\hline Esperar pelo trabalho de outras pessoas, muitas vezes, torna mais lento o ritmo do seu trabalho & 782 & 1,13 & $0,98-1,31$ \\
\hline Seu trabalho é frenético & 702 & 1,32 & $1,14-1,55$ \\
\hline $\begin{array}{l}\text { Suas tarefas muitas vezes, são interrompidas antes que você possa concluí-las, } \\
\text { adiando para mais tarde a sua conclusão }\end{array}$ & 749 & 1,22 & $1,06-1,41$ \\
\hline Seu trabalho exige longos períodos de intensa concentração em uma mesma tarefa & 772 & 1,30 & $1,13-1,50$ \\
\hline Seu trabalho é realizado sob ritmo acelerado & 775 & 1,23 & $1,07-1,41$ \\
\hline O tempo para realização das suas tarefas não é suficiente para conclui-las & 782 & 1,31 & $1,15-1,49$ \\
\hline Você é solicitado a realizar um volume excessivo de trabalho & 769 & 1,40 & $1,23-1,58$ \\
\hline Em seu trabalho, você está livre de solicitações conflitantes & 768 & 1,15 & $0,98-1,35$ \\
\hline \multicolumn{4}{|l|}{ Demanda física } \\
\hline $\begin{array}{l}\text { Muitas vezes, seu trabalho exige que você mantenha seu corpo, por longos períodos, } \\
\text { em posições fisicamente inadequadas e incômodas }\end{array}$ & 783 & 1,38 & $1,13-1,68$ \\
\hline Trabalho exige longos períodos com a cabeça e braço em posição inadequada e incômoda & 762 & 1,28 & $1,07-1,54$ \\
\hline Seu trabalho exige atividade rápida e contínua & 773 & 1,33 & $1,14-1,56$ \\
\hline Seu trabalho exige muito esforço físico & 763 & 1,24 & $1,08-1,42$ \\
\hline Você muitas vezes é solicitado, durante sua jornada de trabalho, a mover ou levantar cargas pesadas & 771 & 1,14 & $0,97-1,33$ \\
\hline
\end{tabular}

controu associação estatística de DPM com a grande maioria das variáveis representativas de demanda e suporte social no trabalho. Entretanto, apenas uma variável relativa ao controle estava associada estatisticamente à DPM, neste mesmo estudo.

A hipótese de Karasek \& Theorell 9 de que o trabalho em alta exigência seria um preditor de maiores riscos à saúde foi confirmada em nosso estudo. Os professores em situação laboral de alta exigência apresentaram elevada prevalência de DPM. Curiosamente, professores em trabalho ativo apresentaram prevalência mais elevada de DPM que os em trabalho passivo. Este mesmo resultado apareceu em enfermeiras de Salvador 10 e em professores da rede particular de Vitória da Conquista 27 , mostrando que um alto controle nessas categorias nem sempre é capaz de diminuir os efeitos negativos à saúde provenientes da alta demanda. O trabalho docente permite ao professor controlar algumas questões inerentes ao seu trabalho, principalmente, as que dizem respeito às questões pedagógicas executadas dentro de sala de aula. Mas, ao mesmo tempo, os resultados mostram que o controle destas questões parece não poupar os educadores das demandas globais a que estão submetidos, como tarefas extras-classe, extensa jornada de trabalho, cumprimento de tarefa com prazo curto de tempo, múltiplos empregos etc. Em nosso estudo, $61,2 \%$ dos professores tinham carga horária docente em sala de aula em todas as escolas igual ou maior que quarenta horas semanais. O somatório destas demandas propicia o surgimento de efeitos sobre a saúde mental deste grupo ocupacional.

Professores no nível intermediário de exposição do modelo de Karasek, trabalho passivo, não apresentaram prevalência de DPM significantemente mais elevada que professores com trabalho de baixa exigência. Esse resultado parece demonstrar que quando a demanda é bai$\mathrm{xa}$, o controle dos docentes tem pouco impacto sobre os distúrbios psíquicos menores.

As menores prevalências dos efeitos estudados foram observadas em trabalho de baixa exigência, ou seja, em situações em que o professor não estava exposto a nenhum dos dois fatores de risco (baixo controle e alta demanda). As diferenças de prevalências entre baixa exigência e trabalho ativo e entre baixa exigência e alta exigência foram estatisticamente sig- 
Tabela 4

Razões de prevalência (RP) e IC95\% para a associação entre distúrbios psíquicos menores e questões relacionadas ao controle do Job Content Questionnaire (JCQ), nos professores da rede municipal de ensino de Vitória da Conquista, Bahia, Brasil, 2001.

\begin{tabular}{|c|c|c|c|}
\hline \multirow[t]{2}{*}{ Questões do JCQ } & \multicolumn{3}{|c|}{ SRQ-20 } \\
\hline & $\mathrm{N}$ & $\mathrm{RP}$ & IC95\% \\
\hline \multicolumn{4}{|l|}{ Controle do trabalho } \\
\hline Baixo controle & 137 & 1,22 & $1,06-1,41$ \\
\hline Alto controle & 547 & 1,00 & - \\
\hline \multicolumn{4}{|l|}{ Habilidades no trabalho } \\
\hline Seu trabalho não requer que você seja criativo & 783 & 1,02 & $0,54-1,95$ \\
\hline Seu trabalho não exige um alto nível de habilidade & 783 & 0,98 & $0,71-1,34$ \\
\hline Seu trabalho não lhe possibilita aprender coisas novas & 785 & 1,20 & $1,02-1,41$ \\
\hline Você não é encarregado de muitas tarefas diferentes & 781 & 0,88 & $0,74-1,04$ \\
\hline Você não tem oportunidade de desenvolver habilidades especiais & 752 & 1,21 & $1,07-1,37$ \\
\hline Seu trabalho é repetitivo & 779 & 1,24 & $1,10-1,40$ \\
\hline \multicolumn{4}{|l|}{ Poder de decisão dentro do grupo } \\
\hline Você não é capaz de dar opinião sobre o que acontece no seu trabalho & 767 & 1,44 & $1,20-1,73$ \\
\hline Seu trabalho não lhe permite tomar decisões sobre as tarefas que você realiza & 779 & 1,23 & $1,02-1,47$ \\
\hline Em seu trabalho você tem pouca liberdade para decidir como fazer suas próprias tarefas & 787 & 1,25 & $1,09-1,43$ \\
\hline \multicolumn{4}{|l|}{ Poder de decisão dentro do clube } \\
\hline Seu grupo de trabalho ou unidade não toma decisões democraticamente & 756 & 1,25 & $1,10-1,43$ \\
\hline Você não tem influência significativa sobre as decisões em seu grupo de trabalho & 757 & 1,32 & $1,17-1,50$ \\
\hline $\begin{array}{l}\text { Há possibilidade de suas idéias não serem consideradas na elaboração } \\
\text { das políticas adotadas na escola }\end{array}$ & 758 & 1,17 & $1,02-1,34$ \\
\hline Você não tem influência sobre as políticas do sindicato de empregados & 649 & 1,28 & $1,10-1,49$ \\
\hline $\begin{array}{l}\text { Sua opinião ou do sindicato não tem influência sobre a política da administração } \\
\text { ou da Secretaria Municipal de Educação e Cultura }\end{array}$ & 627 & 1,09 & $0,95-1,24$ \\
\hline
\end{tabular}

Tabela 5

Razões de prevalência (RP) e IC95\% para a associação entre distúrbios psíquicos menores e questões relacionadas ao suporte social do Job Content Questionnaire (JCQ), nos professores da rede municipal de ensino de Vitória da Conquista, Bahia, Brasil, 2001.

\begin{tabular}{|c|c|c|c|}
\hline \multirow[t]{2}{*}{ Questões do JCQ } & \multicolumn{3}{|c|}{ SRQ-20 } \\
\hline & $\mathrm{N}$ & $\mathrm{RP}$ & IC95\% \\
\hline \multicolumn{4}{|l|}{ Suporte social } \\
\hline Baixo suporte & 319 & 1,27 & $1,13-1,44$ \\
\hline Alto suporte & 471 & 1,00 & - \\
\hline As pessoas com quem você trabalha não são competentes em fazer suas atividades & 774 & 1,18 & $1,00-1,40$ \\
\hline As pessoas no seu trabalho não são amigáveis & 772 & 1,20 & $1,02-1,41$ \\
\hline Seu coordenador ou diretor não presta atenção nas coisas que você fala ou sugere & 765 & 1,34 & $1,18-1,54$ \\
\hline $\begin{array}{l}\text { As pessoas com que você trabalha não são dispostas a colaborar umas com as outras, } \\
\text { na realização das atividades }\end{array}$ & 776 & 1,41 & $1,24-1,56$ \\
\hline Falta de interesse das pessoas do trabalha pelo que acontece com você & 761 & 1,33 & $1,17-1,50$ \\
\hline As pessoas com quem você trabalha não encorajam umas às outras a trabalharem juntas & 768 & 1,27 & $1,12-1,41$ \\
\hline Seu coordenador ou diretor não colabora com você na realização do seu trabalho & 771 & 1,32 & $1,17-1,50$ \\
\hline Falta preocupação do coordenador ou diretor com o bem-estar da sua equipe de trabalho & 771 & 1,24 & $1,08-1,42$ \\
\hline Seu coordenador ou diretor não é bem sucedido em promover o trabalho em equipe & 762 & 1,30 & $1,15-1,47$ \\
\hline Você está exposto a hostilidades e conflitos com as pessoas com quem você trabalha & 766 & 1,24 & $1,09-1,41$ \\
\hline Você está exposto a situações de hostilidades e conflito com seu coordenador ou diretor & 766 & 1,24 & $1,08-1,41$ \\
\hline Indicador de baixo suporte social & 790 & 1,27 & $1,13-1,44$ \\
\hline
\end{tabular}


Razões de prevalência (RP) e respectivos IC95\% para a associação entre distúrbios psíquicos menores (DPM) e conteúdo do trabalho em professores de Vitória da Conquista, Bahia, Brasil, 2001.

\begin{tabular}{|c|c|c|c|c|}
\hline Conteúdo do trabalho & $N^{*}$ & $\begin{array}{l}\text { Prevalência } \\
\text { de DPM (\%) }\end{array}$ & $\begin{array}{l}\text { RP bruta } \\
\text { (IC95\%) }\end{array}$ & $\begin{array}{l}\text { RP ajustada } \\
(I C 95 \%)^{\star \star}\end{array}$ \\
\hline $\begin{array}{l}\text { Baixa exigência } \\
(\downarrow \text { Demanda }+\uparrow \text { Controle) }\end{array}$ & 257 & 45,1 & 1,00 & 1,00 \\
\hline $\begin{array}{l}\text { Trabalho passivo } \\
(\downarrow \text { Demanda }+\downarrow \text { Controle) }\end{array}$ & 56 & 51,8 & $1,15(0,86-1,53)$ & $1,24(0,92-1,66)$ \\
\hline $\begin{array}{l}\text { Trabalho ativo } \\
(\uparrow \text { Demanda }+\uparrow \text { Controle) }\end{array}$ & 255 & 62,4 & $1,38(1,17-1,63)$ & $1,35(1,13-1,61)$ \\
\hline $\begin{array}{l}\text { Alta exigência } \\
(\uparrow \text { Demanda }+\downarrow \text { Controle) }\end{array}$ & 72 & 77,8 & $1,72(1,43-2,07)$ & $1,74(1,44-2,10)$ \\
\hline
\end{tabular}

* A razão de prevalência foi calculada com a situação de baixa exigência no denominador.

** RP ajustada por sexo, vínculo de trabalho, zona de trabalho, carga horária semanal

em sala de aula e recebimento de ajuda em atividade doméstica.

nificantes. Nossos achados apóiam a hipótese de que a saúde dos trabalhadores é preservada nas situações em que as demandas laborais são mantidas em um patamar aceitável e com possibilidade de controle das condições para responder adequadamente a essas demandas.

\section{Resumo}

Um estudo de corte transversal, com todos os professores da rede municipal de ensino de Vitória da Conquista, Bahia, Brasil, investigou a associação entre conteúdo do trabalho (demanda psicológica e controle sobre o trabalho) e a ocorrência de distúrbios psíquicos menores (DPM) entre professores. O Modelo DemandaControle, de Karasek, foi utilizado para avaliar o conteúdo do trabalho. Para mensuração dos distúrbios psíquicos menores utilizou-se o Self-Report Questionnaire-20. A prevalência de DPM foi de $55,9 \%$ entre os 808 professores estudados. A prevalência bruta de DPM mostrou associação positiva e significante com demanda psicológica e associação negativa e significante com controle sobre o trabalho. As prevalencias de DPM foram mais elevadas em professores com trabalho em alta exigência, caracterizado por alta demanda e baixo controle $(R P=1,74$; IC95\%: $1,44-2,10) e$ naqueles em trabalho ativo, com alta demanda e alto controle $(R P=1,35$; IC95\%: 1,13-1,61) quando comparadas à dos professores em trabalho de baixa exigência (baixa demanda e alto controle), depois de ajuste para confundidores num modelo de regressão logística múltipla. Conclui-se que a saúde mental dos professores está fortemente associada ao conteúdo de seu trabalho.

Saúde Mental; Condições de Trabalho; Estresse Psicológico; Transtornos Mentais; Psicologia do Trabalho
Estudos futuros devem investigar a associação entre os efeitos sobre a saúde do docente considerando especialmente o trabalho ativo, uma vez que os achados do nosso estudo revelaram elevadas prevalências de DPM nesse grupo.

\section{Colaboradores}

Todos os autores aprovaram todas as versões do artigo. E. J. F. B. Reis participou da concepção e da coordenação geral da pesquisa, da coleta e análise dos dados e da redação do artigo. T. M. Araújo contribuiu na concepção e coordenação geral da pesquisa, na coleta dos dados e na redação do artigo. F. M. Carvalho participou da coleta de dados e da redação do artigo. L. A. Porto contribuiu na coleta e análise dos dados. A. M. Silvany Neto participou da análise dos dados. 


\section{Referências}

1. Capel SA. The incidence of and influences on stress and burnout in secondary school teachers. Br J Educ Psychol 1987; 57:279-88.

2. Maslach CE, Jackson SE. The measurement of experienced burnout. Journal of Occupational Behavior 1981; 2:99-113.

3. Ministério da Saúde. Doenças relacionadas ao trabalho: manual de procedimentos para os serviços de saúde. Brasília: Ministério da Saúde; 2001.

4. Carlotto MS. Síndrome de Burnout e satisfação no trabalho: um estudo com professores universitários. In: Benevides-Pereira AMT, organizador. Burnout: quando o trabalho ameaça o bem estar do trabalhador. São Paulo: Casa do Psicólogo; 2002. p. 187-212.

5. Carlotto MS. Burnout e o trabalho docente: considerações sobre a intervenção. Revista Eletrônica InterAção Psy 2003; 1:12-8. http://www.dpi. uem.br/Interacao/Numero\%201/PDF/Artigos/Ar tigo2.pdf.

6. Codo W. Educação: carinho e trabalho. Petrópolis: Editora Vozes/Confederação Nacional dos Trabalhadores na Educação; 1999.

7. Araújo TM, Silvany Neto AM, Reis EJFB, Dutra FRD, Azi GR, Alves RL. Trabalho docente e sofrimento psíquico: um estudo entre professores de escolas particulares de Salvador. Revista da FAEEBA 2003; 12:485-95.

8. Delcor NS, Araújo TM, Reis EJFB, Porto L, Carvalho FM, Silva MO, et al. Condições de trabalho e saúde dos professores da rede particular de ensino de Vitória da Conquista, Bahia, Brasil. Cad Saúde Pública 2004; 20:187-203.

9. Karasek R, Theorell T. Healthy work-stress, productivity, and the reconstruction of working life. New York: Basic Books; 1990.

10. Araújo TM, Aquino E, Menezes G, Santos CO, Aguiar L. Aspectos psicossociais do trabalho e distúrbios psíquicos entre trabalhadores de enfermagem. Rev Saúde Pública 2003; 37:424-33.

11. Araújo TM, Graça CC, Araújo E. Estresse ocupacional e saúde: contribuições do Modelo Demanda-Controle. Ciênc Saúde Coletiva 2003; 8:9911003.

12. Araújo TM, Karasek R. Job Content Questionnaire: formal and informal job in a Brazilian study. Int J Behav Med 2004; 11 Suppl:167-8.

13. Alves MGM, Chor D, Faerstein E, Lopes CS, Werneck GL. Versão reduzida da "Job Stress Scale": adaptação para o português. Rev Saúde Pública 2004; 38:164-71.

14. Karasek R. Job Content Questionnaire user's guide. Lowell: University of Massachusetts; 1985.

15. Mari JJ, Williams P. A validity study of psychiatric screening questionnaire (SRQ-20) in primary care in the city of São Paulo. Br J Psychiatry 1986; 148:23-36.
16. Aquino EML. Gênero, trabalho e hipertensão arterial: um estudo de trabalhadoras de enfermagem em Salvador, Bahia [Tese de Doutorado]. Salvador: Universidade Federal da Bahia; 1996.

17. Oliveira NF, Santava VS, Lopes AA. Razões de proporções e uso do método delta para intervalos de confiança em regressão logística. Rev Saúde Pública 1997; 31:90-9.

18. Wernick R. Condições de saúde e trabalho dos docentes da Universidade Federal da Bahia, Salvador, Bahia [Dissertação de Mestrado]. Salvador: Universidade Federal da Bahia; 1999.

19. Cifuentes M. Sintomatologia psiquiátrica según SRQ-20 y factores asociados en profesores municipalizados de la comuna de Talcahuano [Tese de Doutorado]. Concepcíon: Universidad de Concepcíón; 2000.

20. Oliveira MG. Condições de trabalho, gênero e saúde: sofrimento e estresse. Um estudo de caso com os profissionais docente do ensino superior privado de Belo Horizonte [Dissertação de Mestrado]. Belo Horizonte: Pontifícia Universidade Católica de Minas Gerais; 2001.

21. Paranhos I. Interface entre trabalho docente e saúde dos professores da Universidade Estadual de Feira de Santana [Dissertação de Mestrado]. Feira de Santana: Universidade Estadual de Feira de Santana; 2001.

22. Borges $\mathrm{CH}$. Transtornos mentais menores entre trabalhadores de uma usina siderúrgica [Dissertação de Mestrado]. São Paulo: Universidade de São Paulo; 1990.

23. Fernandes SNP. Trabalho informativo e distúrbios psíquicoemocionais: estudo seccional em três empresas de processamento de dados de Salvador - BA [Dissertação de Mestrado]. Salvador: Universidade Federal da Bahia; 1992.

24. Pitta A. Hospital, dor e morte como ofício. São Paulo: Editora Hucitec; 1990.

25. Faria NMX, Facchini LA, Fassa AG, Tomasi E. Estudo transversal sobre saúde mental de agricultores da Serra Gaúcha (Brasil). Rev Saúde Pública 1999; 33:391-400.

26. Ludemir AB, Melo Filho DA. Condições de vida e estrutura ocupacional associadas a transtornos mentais comuns. Rev Saúde Pública 2002; 36:21321.

27. Delcor NS. Condições de trabalho e saúde dos professores da rede particular de ensino em Vitória da Conquista - BA [Dissertação de Mestrado]. Salvador: Universidade Federal da Bahia; 2003.

Recebido em 29/Set/2004

Versão final reapresentada em 09/Mar/2005 Aprovado em 18/Abr/2005 